\title{
Stroke: A Brain Attack
}

\author{
Guide- Miss.B.M.Gund $^{1 *}$, Mrs.P.N.Jagtap ${ }^{1}$, Mr.V.B.Ingale ${ }^{1}$, Dr.R.Y.Patil ${ }^{1}$ \\ Shankarrao ursal college of Pharmaceutical science and research centre Kharadi, Pune \\ College: - S.U.C.O.Pharmaceutical Science \& Research Centre Kharadi, Pune
}

\begin{abstract}
There are 15 million people worldwide who suffer a stroke each year. According to the World Health Organization (WHO), stroke is the second leading cause of death for people above the age of 60 years, and the fifth leading cause in people aged 15 to 59 years old. Each year, nearly six million people worldwide die from stroke. One in six people worldwide will have a stroke in their lifetime. Every six seconds, stroke kills some. Stroke claims more than twice as many lives as AIDS. In fact, stroke continues to be responsible for more deaths annually than those attributed to AIDS, tuberculosis and malaria combined. The burden of stroke now disproportionately affects individuals living in resource-poor countries.Stroke, as a burden of disease, still remains little understood. Enhanced advocacy and sustained public health education at the global level must be undertaken. To date, stroke education and the delivery of the best possible care for stroke survivors needs to be put higher up on the public health agenda. A passive attitude towards stroke is no longer justifiable: stroke can be potentially prevented to a large extent, and acute stroke is potentially treatable. Notwithstanding, there is much more that can be done to improve the quality of life for people who have suffered from a stroke. Without coordinated effort on the part of the international public health community, stroke will claim the lives of up to 6.5 million people each year by 2015, according to the World Stroke Organization.
\end{abstract}

KEYWORDS : tPA(Tissue plasminogen activator), CVA(Cerebral vascular accident), TIA(Transient ischemic attack, TNK(Tenecteplase), Rehabilitation, Aneurysms.

\section{INTRODUCTION}

Stroke, also called brain attack, is a brain injury caused by a sudden interruption in the blood supply of the brain. It occurs when part of the brain does not receives the needed blood flow for one of two reasons either the blood supply to part of the brain is suddenly interrupted, or because a blood vessel in the brain ruptures and blood invades the surrounding areas. The brain is the central information-processing organ of the body responsible with the control of multiple complex functions. Without the blood supply, the brain cells start to die (cerebral infarction) leading to brain damage. Approximately two million brain cells die every minute during a stroke which increases the risk of brain damage, disability, and death. The level of disability varies from patient to patient according to the type of stroke suffered, the part of the brain affected, and the size of the damaged area.Stroke is the third leading causes of death in United States and UK after heart disease and cancer, and the number one cause of adult disability. In the United States, over 160,000 American adults die of stroke each year. In Europe, approximately 650,000 people die of stroke. In the United States, the majority of people that have a stroke are over the age of 65 . However, 28 percent of all stroke cases occur in people younger than 65. The risk of having a stroke more than doubles each decade after the age of 55.A stroke is a medical emergency that can affect individuals of all age categories including children and babies. Among adults age 45 to 65,8 to 12 percent of the ischemic stroke cases and 37 to 38 percent of hemorrhagic stroke cases result in death within 30 days. Within a year, 25 percent of those that had a transient ischemic attack will die, and this percentage increases among seniors 65 and older.On average in the United States, someone suffers a stroke every 45 seconds, and at every 3 to 4 minutes someone dies of a stroke. Every seven minutes a person dies of heart disease or stroke in Canada, while every five minutes somebody suffers a stroke in the UK.According to the World Health Organization, 15 million people worldwide have a stroke ever year, of which 5 million die and 5 million are permanently disabled. However, medical research shows that every year 80 percent of the all cases of stroke can be prevented. ${ }^{[1]}$

\section{HISTORY}

Episodes of stroke and familial stroke have been reported from the 2nd millennium BC onward in ancient Mesopotamia and Persia. Hippocrates (460 to $370 \mathrm{BC}$ ) was first to describe the phenomenon of sudden paralysis that is often associated with ischemia. Apoplexy, from the Greek word meaning "struck down with violence," first appeared in Hippocratic writings to describe this phenomenon. 
The word stroke was used as a synonym for apoplectic seizure as early as 1599 , and is a fairly literal translation of the Greek term.In 1658, in his Apoplexia, Johann Jacob Wepfer (1620-1695) identified the cause of hemorrhagic stroke when he suggested that people who had died of apoplexy had bleeding in their brains. Wepfer also identified the main arteries supplying the brain, the vertebral and carotid arteries, and identified the cause of ischemic stroke [also known as cerebral infarction] when he suggested that apoplexy might be caused by a blockage to those vessels. Rudolf Virchow first described the mechanism of thromboembolism as a major factor. Hippocrates, the father of medicine, first recognized stroke over 2,400 years ago. At this time stroke was called apoplexy, which means "struck down by violence" in Greek. This was due to the fact that a person developed sudden paralysis and change in well-being. Physicians had little knowledge of the anatomy and function of the brain, the cause of stroke, or how to treat it.It was not until the mid-1600s that Jacob Wepfer found that patients who died with apoplexy had bleeding in the brain. He also discovered that a blockage in one of the brain's blood vessels could cause apoplexy.Medical science continued to study the cause, symptoms, and treatment of apoplexy and, finally, in 1928, apoplexy was divided into categories based on the cause of the blood vessel problem. This led to the terms stroke or "cerebral vascular accident (CVA)." Stroke is now often referred to as a "brain attack" to denote the fact that it is caused by a lack of blood supply to the brain, very much like a "heart attack" is caused by a lack of blood supply to the heart. The term brain attack also conveys a more urgent call for immediate action and emergency treatment by the general public.Today, there is a wealth of information available on the cause, prevention, risk, and treatment of stroke. Although there is no cure, most stroke victims now have a good chance for survival and recovery. Immediate treatment, supportive care, and rehabilitation can all improve the quality of life for stroke victims. ${ }^{[2]}$

\section{EPIDEMIOLOGY OF THE STROKE}

Global measurements undertaken by the WHO revealed an up to ten-fold difference in age-adjusted and sex-adjusted mortality rates and burden (measured in disability-adjusted life year loss rates (DALYs)) among countries. Both were considerably higher in low-income countries (North Asia, Eastern Europe, Central Africa, and South Pacific) compared to high-income countries (Western Europe, North America). ${ }^{[3]}$

\subsection{Prevalence}

- One in every 10 deaths is caused by stroke; thus it is the third most common cause of death in developed countries, exceeded only by coronary heart disease and cancer.

- The prevalence of stroke in the US is about 7 million (3.0\%).

- In China, the prevalence of stroke ranges between 1.8\% (rural areas) and 9.4\% (urban areas).

- Worldwide, China has one of the highest rates of mortality (19.9\% of all deaths in China), along with Africa and parts of South America. ${ }^{[4]}$

\subsection{Incidence}

- Worldwide, 15 million people suffer a stroke each year; one-third die and one-third are left permanently disabled.

- 795,000 new or recurrent strokes occur per year in the US, accounting for approximately 1 in 18 deaths.

- In Europe, the incidence of stroke varies from 101.1 to 239.3 per 100,000 in men and 63.0 to 158.7 per 100,000 in women.

- Within 5 years of a stroke, over half of patients aged $\geq 45$ years will die: $52 \%$ of men and $56 \%$ of women. (see figure 1)

\subsection{Cost of Stroke}

- The estimated direct and indirect cost of stroke in the US for 2010 was $\$ 73.7$ billion.

- The estimated cost of stroke in Europe in 2010 was approximately $€ 64.1$ billion. $^{[5]}$ 


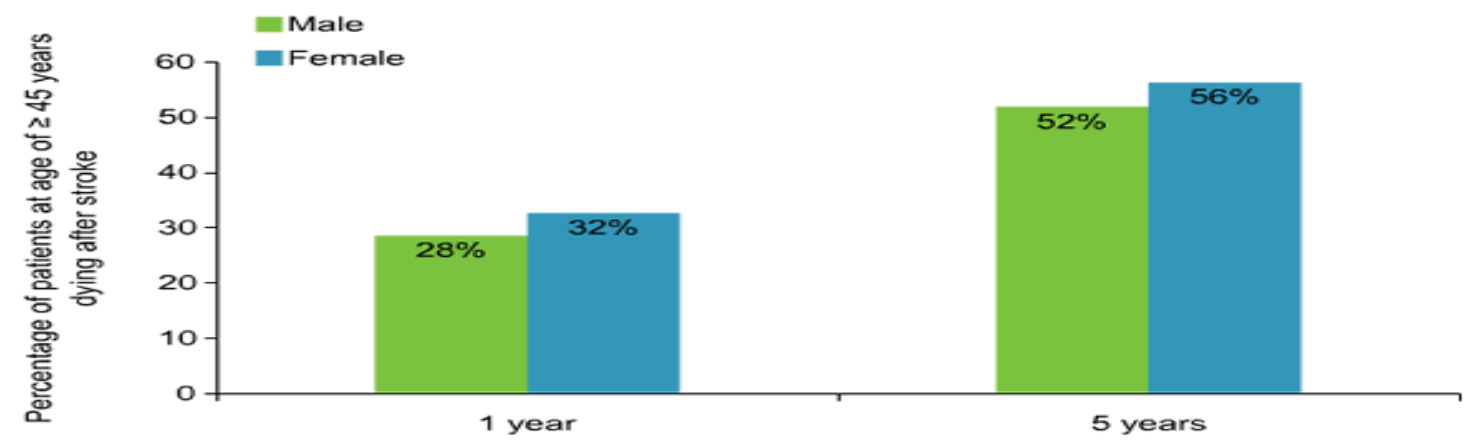

“Fig1": Mortality following a stroke

"Fig 2": Stroke

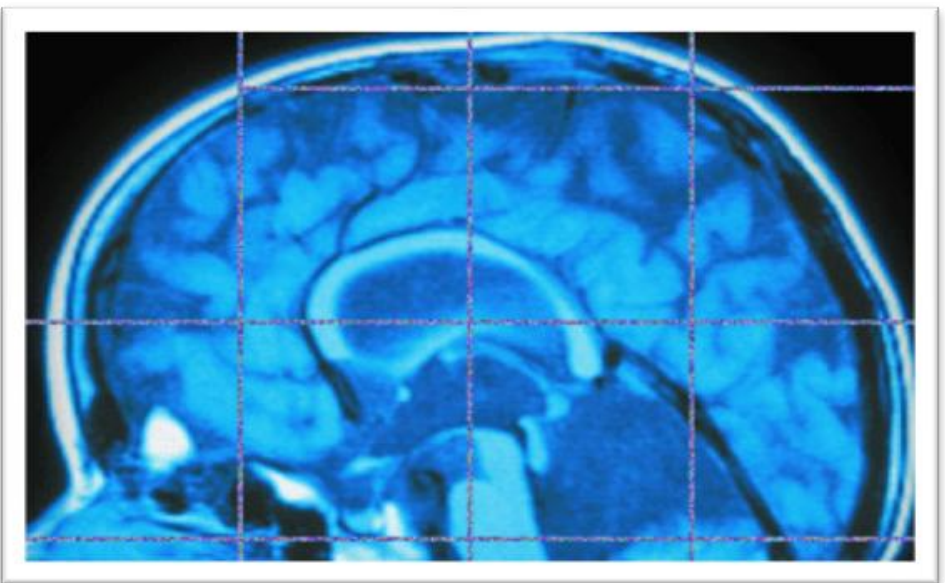

IV. WHAT IS STROKE?

Stroke is the fourth leading cause of death in World and a leading cause of adult disability. Up to $80 \%$ of strokes are preventable; you can prevent a stroke!A stroke or "brain attack" occurs when a blood clot blocks an artery (a blood vessel that carries blood from the heart to the body) or a blood vessel (a tube through which the blood moves through the body) breaks, interrupting blood flow to an area of the brain. When either of these things happen, brain cells begin to die and brain damage occurs. When brain cells die during a stroke, abilities controlled by that area of the brain are lost. These abilities include speech, movement and memory. How a stroke patient is affected depends on where the stroke occurs in the brain and how much the brain is damaged.

For example, someone who has a small stroke may experience only minor problems such as weakness of an arm or leg. People who have larger strokes may be paralyzed on one side or lose their ability to speak. Some people recover completely from strokes, but more than $2 / 3$ of survivors will have some type of disability. 


\section{TYPES OF STROKE}

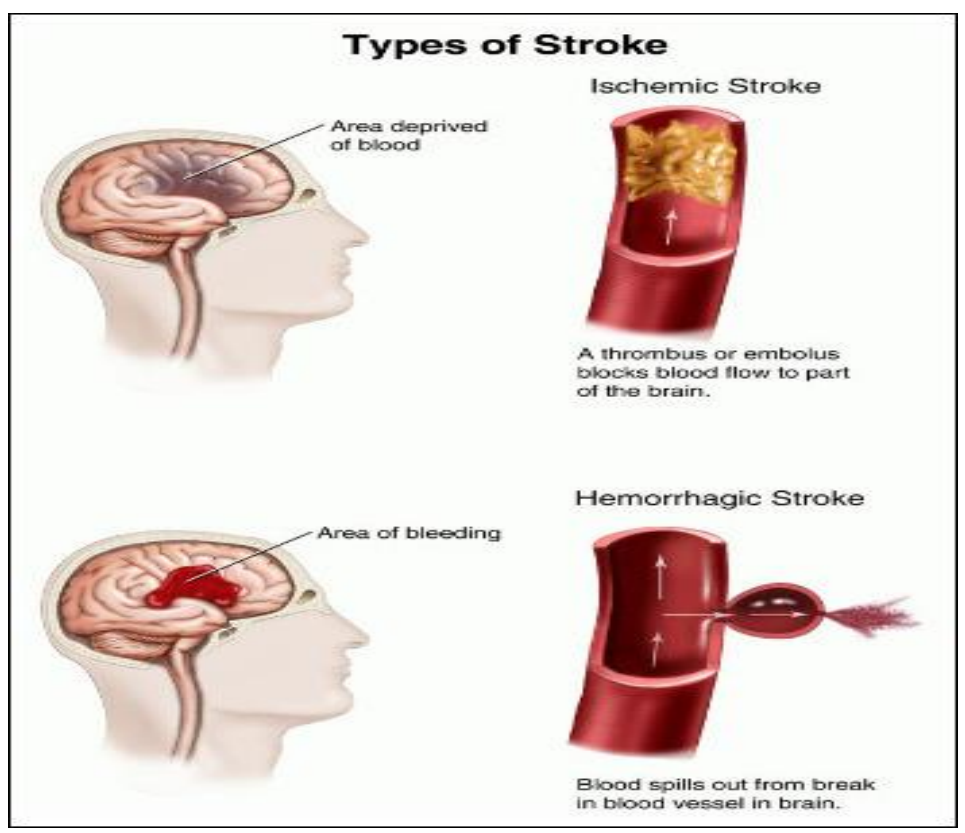

“Fig3": Types of Stroke

The two major class of strokes

\subsection{Ischemic Stroke}

In everyday life, blood clotting is beneficial. When you are bleeding from a wound, blood clots work to slow and eventually stop the bleeding. In the case of stroke, however, blood clots are dangerous because they can block arteries and cut off blood flow, a process called ischemia. An ischemic stroke can occur in two ways: embolic and thrombotic strokes ${ }^{[6]}$

\subsection{1 - Embolic Stroke}

In an embolic stroke, a blood clot forms somewhere in the body (usually the heart) and travels through the bloodstream to your brain. Once in your brain, the clot eventually travels to a blood vessel small enough to block its passage. The clot lodges there, blocking the blood vessel and causing a stroke. The medical word for this type of blood clot is embolus.

\subsection{2- Thrombotic Stroke}

In the second type of blood-clot stroke, blood flow is impaired because of a blockage to one or more of the arteries supplying blood to the brain. The process leading to this blockage is known as thrombosis. Strokes caused in this way are called thrombotic strokes. That's because the medical word for a clot that forms on a blood-vessel deposit is thrombus. Blood-clot strokes can also happen as the result of unhealthy blood vessels clogged with a buildup of fatty deposits and cholesterol. Your body regards these buildups as multiple, tiny and repeated injuries to the blood vessel wall. So your body reacts to these injuries just as it would if you were bleeding from a wound; it responds by forming clots.Two types of thrombosis can cause stroke: large vessel thrombosis and small vessel disease (or lacunar infarction.)

\subsubsection{1- Large Vessel Thrombosis}

Thrombotic stroke occurs most often in the large arteries, so large vessel thrombosis is the most common and best understood type of thrombotic stroke. Most large vessel thrombosis is caused by a combination of long-term atherosclerosis followed by rapid blood clot formation. Thrombotic stroke patients are also likely to have coronary artery disease, and heart attack is a frequent cause of death in patients who have suffered this type of brain attack. 


\subsubsection{2- Small Vessel Disease/Lacunar Infarction}

Small vessel disease, or lacunar infarction, occurs when blood flow is blocked to a very small arterial vessel. The term's origin is from the Latin word lacuna which means hole, and describes the small cavity remaining after the products of deep infarct have been removed by other cells in the body. Little is known about the causes of small vessel disease, but it is closely linked to hypertension (high blood pressure).

\section{3- Hemorrhagic Stroke}

Strokes caused by the breakage or "blowout" of a blood vessel in the brain are called hemorrhagic strokes. The medical word for this type of breakage is hemorrhage. Hemorrhages can be caused by a number of disorders which affect the blood vessels, including long-standing high blood pressure and cerebral aneurysms. An aneurysm is a weak or thin spot on a blood vessel wall. These weak spots are usually present at birth. Aneurysms develop over a number of years and usually don't cause detectable problems until they break. There are two types of hemorrhagic stroke: subarachnoid and intracerebral.In an intracerebral hemorrhage, bleeding occurs from vessels within the brain itself. Hypertension (high blood pressure) is the primary cause of this type of hemorrhage.In a subarachnoid hemorrhage, an aneurysm bursts in a large artery on or near the thin, delicate membrane surrounding the brain. Blood spills into the area around the brain, which is filled with a protective fluid, causing the brain to be surrounded by blood-contaminated fluid.

\section{4- Other forms of stroke}

In addition to the major causes of stroke described above, there are a number of other causes, including the two most common ones: cardiac arrest and hematomas adjacent to the brain. In cardiac arrest, the heart stops pumping blood or does not pump effectively, and the brain is deprived of both oxygen and glucose. Although the entire brain is affected, certain areas are more vulnerable. Memory and coordination are among the most frequent deficits after this type of stroke .Hematomas accumulations of blood that are the result of hemorrhage sometimes occur in the outermost covering of the brain, the subdural or epidural layers. These are usually caused by injury, but may occur spontaneously, especially in the elderly. In this type of stroke, surgery can usually correct the problem by removing the clot, and maybe lifesaving.

\section{SIGN AND SYMPTOMS}

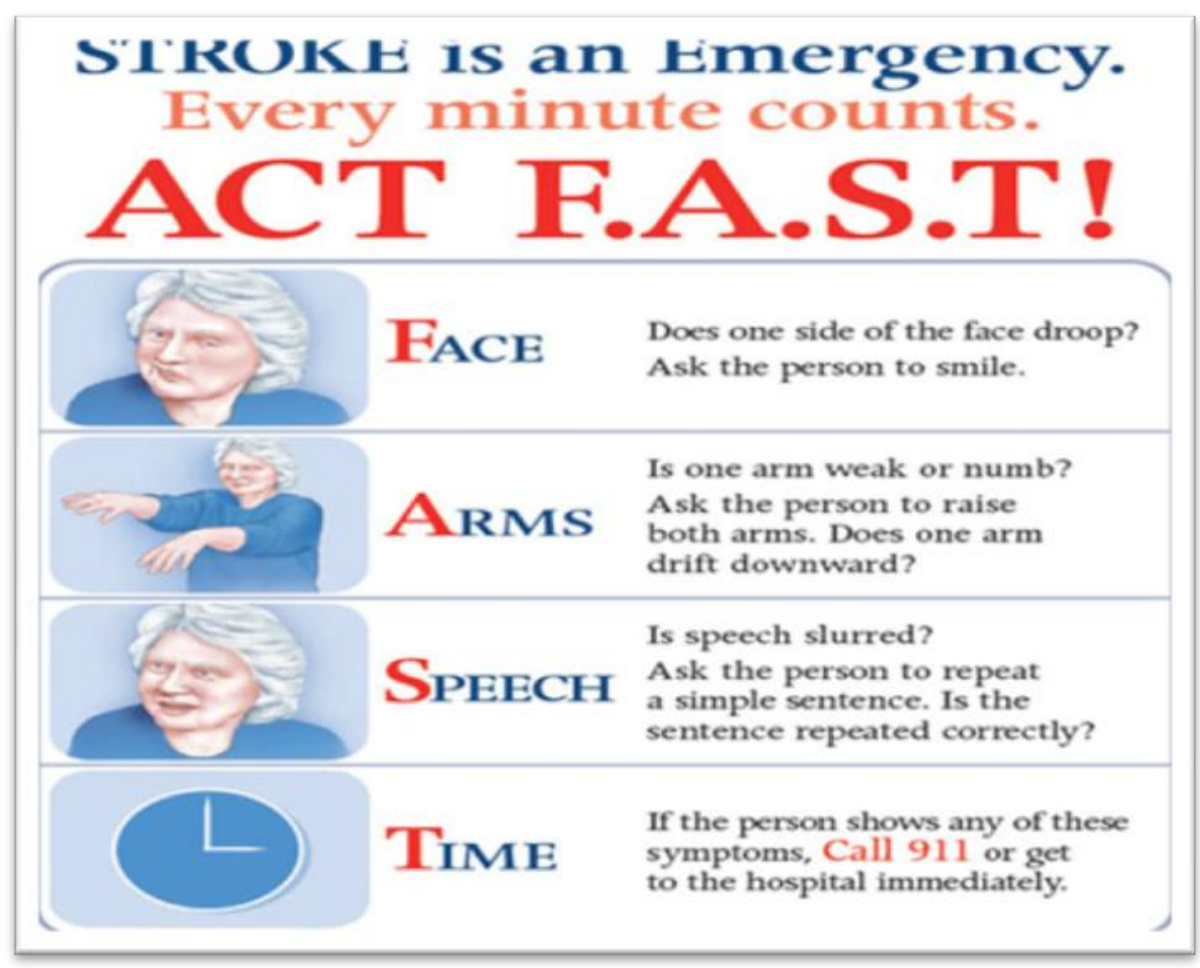

"Fig4": Warning Signs of Stroke 
The symptoms of stroke depend on what part of the brain is damaged. In some cases, a person may not even be aware that he or she has had a stroke. Symptoms usually develop suddenly and without warning, or they may occur on and off for the first day or two. Symptoms are usually most severe when the stroke first happens, but they may slowly get worse. A headache may occur, especially if the stroke is caused by bleeding in the brain. The headache: ${ }^{[10]}$

- $\quad$ Starts suddenly and may be severe

- $\quad$ Occurs when lying flat

- $\quad$ Wakes you up from sleep

- Gets worse when you change positions or when you bend, strain, or cough

Other symptoms depend on the severity of the stroke and what part of the brain is affected. Symptoms may include:

- Change in alertness (including sleepiness, unconsciousness, and coma)

- Changes in hearing

- $\quad$ Changes in taste

- Clumsiness

- $\quad$ Confusion or loss of memory

- Difficulty swallowing

- Difficulty writing or reading

- Dizziness or abnormal sensation of movement (vertigo)

- $\quad$ Lack of control over the bladder or bowels

- $\quad$ Loss of balance

- Loss of coordination

- $\quad$ Muscle weakness in the face, arm, or leg (usually just on one side)

- Numbness or tingling on one side of the body

- $\quad$ Personality, mood, or emotional changes

- $\quad$ Problems with eyesight, including decreased vision, double vision, or total loss of vision

- Sensation changes that affect touch and the ability to feel pain, pressure, different temperatures, or other stimuli

Trouble speaking or understanding others who are speaking

- $\quad$ Trouble walking

\section{PATHOPHYSIOLOGY}

A stroke occurs when the blood flow to an area of the brain is interrupted, resulting in some degree of permanent neurological damage. The two major categories of stroke are ischemic (lack of blood and hence oxygen to an area of the brain) and haemorrhagic (bleeding from a burst or leaking blood vessel in the brain) stroke. ${ }^{[15]}$

\subsection{Pathophysiology of ischemic stroke}

The common pathway of ischemic stroke is lack of sufficient blood flow to perfuse cerebral tissue, due to narrowed or blocked arteries leading to or within the brain. Ischemic strokes can be broadly subdivided into thrombotic and embolic strokes. Narrowing is commonly the result of atherosclerosis - the occurrence of fatty plaques lining the blood vessels. As the plaques grow in size, the blood vessel becomes narrowed and the blood flow to the area beyond is reduced. Damaged areas of an atherosclerotic plaque can cause a blood clot to form, which blocks the blood vessel - a thrombotic stroke. In an embolic stroke, blood clots or debris from elsewhere in the body, typically the heart valves, travel through the circulatory system and block narrower blood vessels. Based on the aetiology of ischemic stroke, a more accurate sub-classification is generally used:

- Large artery disease - atherosclerosis of large vessels, including the internal carotid artery, vertebral artery, basilar artery, and other major branches of the Circle of Willis.

- Small vessel disease - changes due to chronic disease, such as diabetes, hypertension, hyperlipidaemia, and smoking, that lead decreased compliance of the arterial walls and/or narrowing and occlusion of the lumen of smaller vessels.

- Embolic stroke - the most common cause of an embolic stroke is atrial fibrillation.

- Stroke of determined etiology - such as inherited diseases, metabolic disorders, and coagulopathies.

- Stroke of undetermined etiology - after exclusion of all of the above. 
In the core area of a stroke, blood flow is so drastically reduced that cells usually cannot recover and subsequently undergoing cellular death. The tissue in the region bordering the infarct core, known as the ischaemic penumbra, is less severely affected. This region is rendered functionally silent by reduced blood flow but remains metabolically active. Cells in this area are endangered but not yet irreversibly damaged. They may undergo apoptosis after several hours or days but if blood flow and oxygen delivery is restored shortly after the

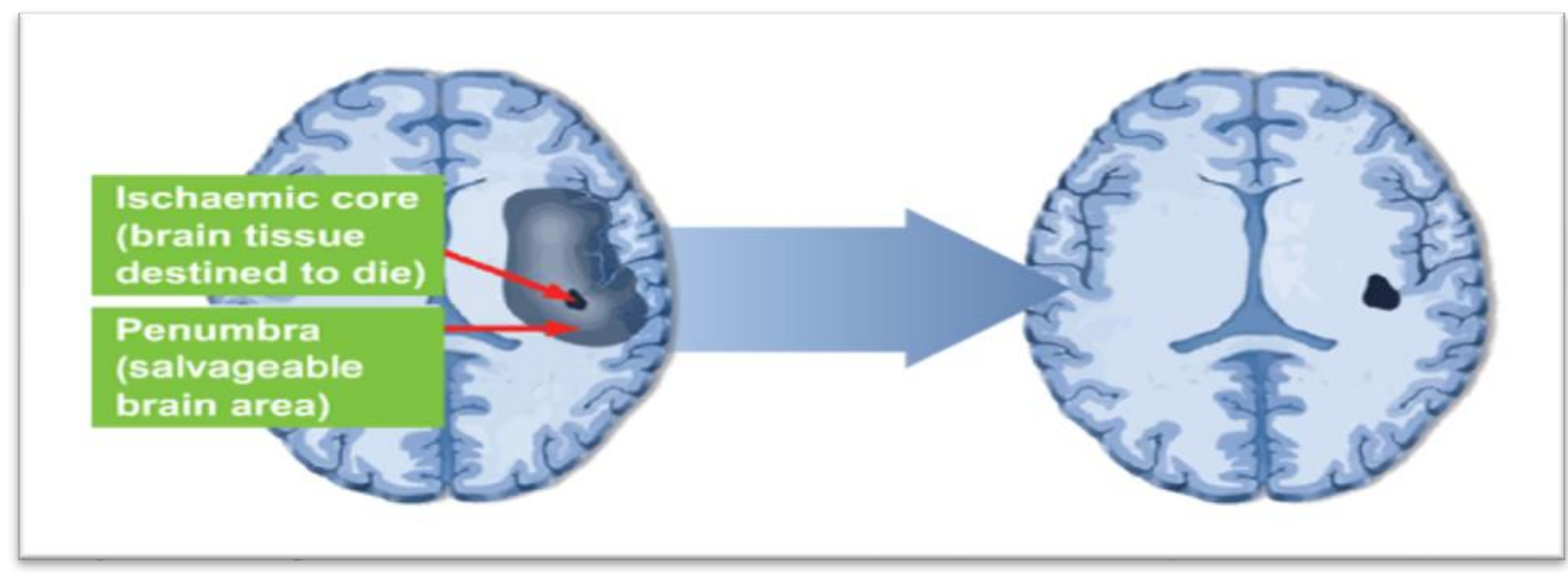

The goal of acute stroke therapy is to normalize perfusion and intervene in the cascade of biochemical

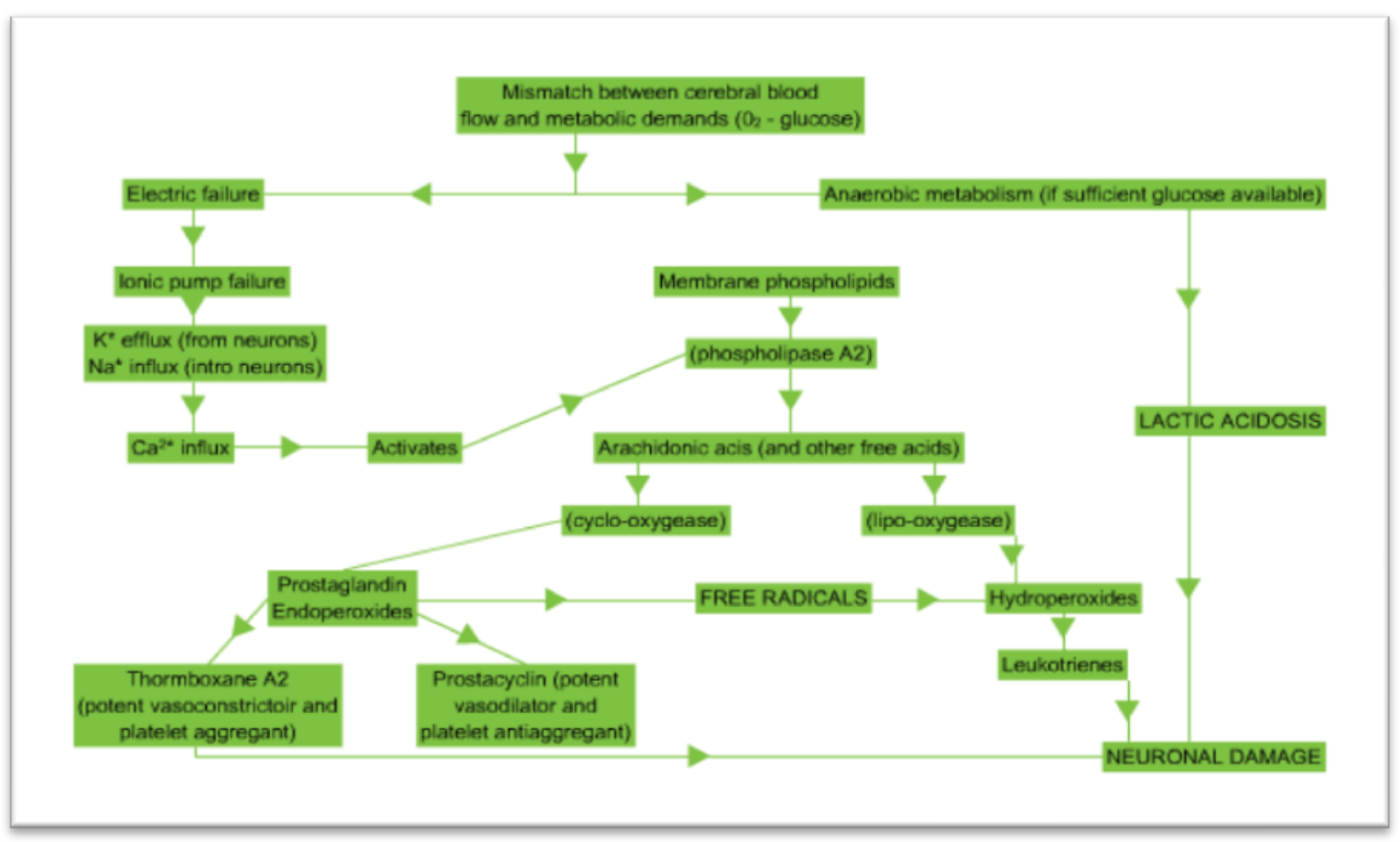

dysfunction to salvage the penumbra as much and as early as possible.

"Fig6": The ischemic cascade

\section{Important steps of the ischemic cascade}

1. Without adequate blood supply and thus lack of oxygen, brain cells lose their ability to produce energy particularly adenosine triphosphate (ATP).

2. Cells in the affected area switch to anaerobic metabolism, which leads to a lesser production of ATP but releases a by-product called lactic acid. 
3. Lactic acid is an irritant, which has the potential to destroy cells by disruption of the normal acid-base balance in the brain.

4. ATP-reliant ion transport pumps fail, causing the cell membrane to become depolarized; leading to a large influx of ions, including calcium (Ca++), and an efflux of potassium.

5. Intracellular calcium levels become too high and trigger the release of the excitatory amino acid neurotransmitter glutamate.

6. Glutamate stimulates AMPA receptors and Ca++-permeable NMDA receptors, which leads to even more calcium influx into cells.

7. Excess calcium entry overexcites cells and activates proteases (enzymes which digest cell proteins), lipases (enzymes which digest cell membranes) and free radicals formed as a result of the ischemic cascade in a process called excitotoxicity.

8. As the cell's membrane is broken down by phospholipases, it becomes more permeable, and more ions and harmful chemicals enter the cell.

9. Mitochondria break down, releasing toxins and apoptotic factors into the cell.

10. Cells experience apoptosis.

11. If the cell dies through necrosis, it releases glutamate and toxic chemicals into the environment around it. Toxins poison nearby neurons, and glutamate can overexcite them.

12. The loss of vascular structural integrity results in a breakdown of the protective blood brain barrier and contributes to cerebral oedema, which can cause secondary progression of the brain injury.

\subsection{Pathophysiology of hemorrhagic stroke}

Hemorrhagic strokes are due to the rupture of a blood vessels leading to compression of brain tissue from an expanding hematoma. This can distort and injure tissue. In addition, the pressure may lead to a loss of blood supply to affected tissue with resulting infarction, and the blood released by brain hemorrhage appears to have direct toxic effects on brain tissue and vasculature.

- Intracerebral hemorrhage - caused by rupture of a blood vessel and accumulation of blood within the brain. This is commonly the result of blood vessel damage from chronic hypertension, vascular malformations, or the use medications associated with increased bleeding rates, such as anticoagulants, thrombolytics, and antiplatelet agents.

- Subarachnoid hemorrhage is the gradual collection of blood in the subarachnoid space of the brain dura, typically caused by trauma to the head or rupture of a cerebral aneurysm

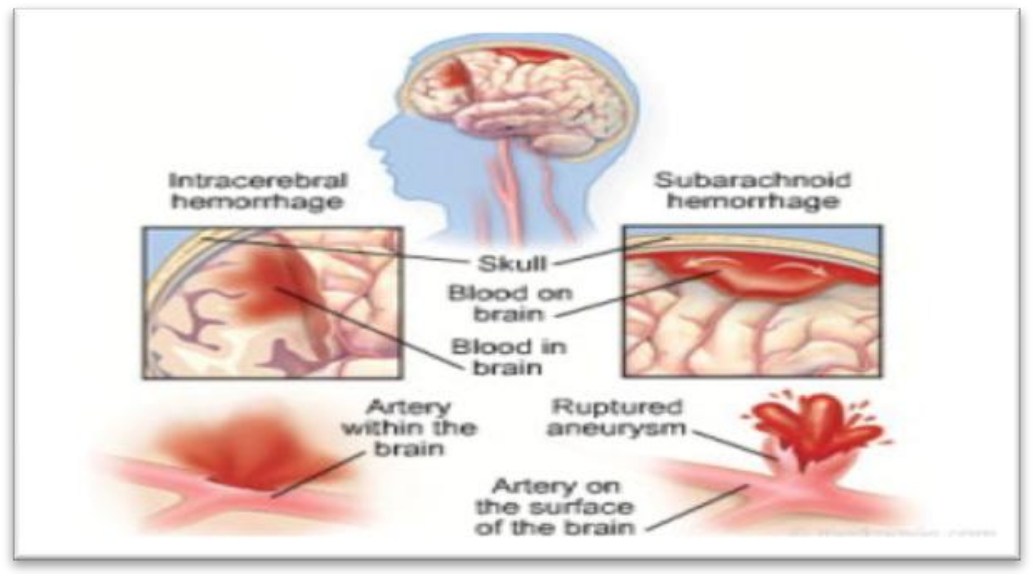

"Fig 7": Pathophysiology of Hemorrhagic Stroke

\section{RISK FACTOR OF STROKE}

There are 2 types of risk factors for stroke: controllable and uncontrollable. Controllable risk factors generally fall into two categories: lifestyle risk factors or medical risk factors. Lifestyle risk factors can often be changed, while medical risk factors can usually be treated. Both types can be managed best by working with a doctor, who can prescribe medications and advise on how to adopt a healthy lifestyle. Uncontrollable risk factors include being over age 55, being male, being African American, Hispanic or Asian/Pacific Islander, or having a family history of stroke or transient ischemic attack (TIA). ${ }^{[21]}$ 


\subsection{Controllable Risk Factors:}

- High Blood Pressure

- Atrial Fibrillation

- High Cholesterol

- Diabetes

- Atherosclerosis

- Circulation Problems

- Tobacco Use and Smoking

- Alcohol Use

- Physical Inactivity

- Obesity

Treatable Disease that Increase Stroke Risk:

High Blood Pressure (Hypertension)

High blood pressure is a major risk factor for stroke. Blood pressure by definition is the force of blood pushing against the walls of your arteries. High blood pressure causes the heart to pump harder to move blood through the body. This can weaken blood vessels and damage major organs such as the brain. Left untreated, high blood pressure can lead to stroke.

\section{Atrial Fibrillation (AF)}

Atrial fibrillation (AF) is caused when the two upper chambers of the heart (atria) beat rapidly and unpredictably, producing an irregular heartbeat. AF raises stroke risk because it allows blood to pool in the heart. When blood pools, it tends to form clots which can then be leading to heart failure.

\section{High Cholesterol}

Cholesterol is a fatty substance in the blood that the human body makes on its own, but it also comes from fat in foods. High levels of cholesterol in the bloodstream can clog arteries and cause a stroke or heart attack.

\section{Diabetes}

In people with diabetes, the body either doesn't produce enough insulin or the cells ignore the insulin. Without insulin, the body can't process sugar, which is the basic fuel for the cells in the body. People with diabetes are up to 4 times more likely to have a stroke than someone who does not have the disease, mainly because many people with diabetes have health problems that are also stroke risk factors.

\section{Atherosclerosis}

Atherosclerosis is the progressive buildup of plaque fatty deposits and other cells in artery walls. It can clog arteries and block the flow of blood to the brain or other parts of the body, making a person more at risk for a stroke, TIA or other heart disease.

\section{Lifestyle Risk Factors That Can Be Changed: \\ Tobacco Use/Smoking}

Among other things, smoking damages blood vessel walls, speeds up the clogging of arteries, raises blood pressure and makes the heart work harder. Smoking also doubles the risk of stroke.

Alcohol Use

Alcohol use has been linked to stroke in many studies. For example, drinking large amounts of alcohol may increase risk for stroke.

\section{Obesity}

Obesity and excess weight put a strain on the entire circulatory system. Obesity also makes people more likely to have high cholesterol, high blood pressure and diabetes all of which can increase risk for stroke. Adopting healthy eating habits and increasing physical activity can help reduce stroke risk. 
8.2 Uncontrollable Risk Factors:

- Age

- Gender

- Race

- Family History

- PreviousFibromuscular Dysplasia

- $\quad$ Patent Foramen Ovale (PFO or Hole in the Heart)

- $\quad$ Stroke or TIA

Age

A stroke can happen to anyone, but risk of stroke increases with age. After the age of 55, stroke risk doubles for every decade a person is alive.

\section{Gender}

Women suffer more strokes each year than men, mainly because women live longer than men and stroke occurs more often at older ages. Annually, about 55,000 more women than men have strokes, but stroke incidence is higher in men than women at younger ages. Additionally, women are two times more likely to die of a stroke than breast cancer annually.A 2006 study of women ages 45 and older showed significantly reduced risk for ischemic stroke (stroke caused by a clot) when women maintained a healthy lifestyle that includes no smoking, moderate alcohol use, average weight for their height, regular exercise and a healthy diet.

Race

African Americans have twice the risk of stroke when compared to Caucasians. Hispanic and Asian/Pacific Islanders also have higher risk than Caucasians.

Family.history

if a family member has had a stroke; everyone in the family has a higher risk of stroke.

\section{Previous stroke or TIA}

after experiencing a stroke, survivors and their families usually concentrate their efforts on rehabilitation and recovery. However, preventing a "recurrent" stroke from happening is also a critical consideration. About 5 to 14 percent of the people who have a stroke this year will have a second one. Within the next 5 years, stroke will recur in 24 percent of women and 42 percent of men.Stroke prevention is also important to those who have experienced transient ischemic attacks (TIAs). TIAs are brief episodes of strokelike symptoms that can last from a few minutes to 24 hours, but usually cause no permanent damage or disability. TIAs are serious warning signs of an impending stroke. Up to 40 percent of people who experience a TIA are expected to have a stroke. However, many recurrent strokes and TIAs can be prevented through lifestyle changes, surgery, medication or a combination of all three methods. FMD is a medical disorder where some of the arteries that carry blood throughout the body do not develop as they should. Fibrous tissue grows in the wall of the arteries, causing them to narrow. As a result, blood flow through the arteries decreases.

Hole in the heart: patent foramen ovale (PFO)

Strokes and TIAs can occur without any obvious risk factors because they are caused by a "hole" in the heart called a patent foramen ovale (PFO). About 1 in 5 Americans has a PFO. Many don't know it until a medical condition like a stroke or TIA occurs. PFOs often have no symptoms but they may increase your risk for stroke and TIA. Many PFO-related strokes are called cryptogenic, meaning they have no apparent cause. ${ }^{\text {[20] }}$

\subsection{What is TIA?}

\section{TRANSIENT ISCHEMIC ATTACK (TIA)}

A transient ischemic attack (TIA) is an event, sometimes called a mini-stroke, with stroke symptoms that last less than 24 hours before disappearing. While TIAs generally do not cause permanent brain damage, they are a serious warning sign of stroke and should not be ignored! Check. Once you calculate your risk, scroll down to see your results .Up to 40 percent of all people who have experienced a TIA will go on to have an actual stroke. Most studies show that nearly half of all strokes occur within the first two days after a TIA. In fact: Within two days after a TIA, 5 percent of people will have a stroke. Within three months after a TIA, 10 to 15 percent of people will have a stroke. 


\subsection{What are the symptoms of a TIA?}

The symptoms of a TIA and stroke are basically the same. Someone having a TIA or stroke may experience one or more of the following symptoms:

- Sudden numbness or weakness of the face, arm or leg, especially on one side of the body

- Sudden confusion, trouble speaking or understanding

- Sudden trouble seeing in one or both eyes

- Sudden trouble walking, dizziness loss of balance or coordination

If you have any of these symptoms or see them in someone else, even for a short time, call 9-1-1 or have someone take you to the hospital immediately. Treatment can be more helpful if given quickly. Stroke strikes FAST. You should too!

\subsection{What causes a TIA?}

Blood vessels carry blood throughout the body. When a blood vessel in the brain becomes blocked for a short period of time, the blood flow to that area of the brain slows or stops. This lack of blood (and oxygen) often leads to temporary symptoms such as slurred speech or blurry vision.

TIAs are usually caused by one of three things:

- Low blood flow at a narrow part of a major artery carrying blood to the brain, such as the carotid artery

- A blood clot in another part of the body (such as the heart) breaks off, travels to the brain and blocks a blood vessel in the brain

- Narrowing of the smaller blood vessel in the brain, blocking blood flow for a short period of time; usually caused by plaque (a fatty substance) build-up

\subsection{How can TIA be managed?}

The goal of TIA management is to prevent a future stroke. The medicine and therapy used depends on the exact cause of the TIA. In addition to lifestyle changes such as diet and exercise, your doctor may recommend drugs to treat high blood pressure, high cholesterol or heart disease. These changes may reduce your risk of further TIA or stroke.There are many medications that help prevent blood clots from forming, reducing the risk of full-blown stroke.If a TIA is caused by blockage in the main artery in the neck that supplies blood to the brain, called the carotid artery, surgeries may be required to open the artery and prevent a stroke. These procedures are known as endarterectomy and stenting. Talk to a doctor about the best stroke prevention options for you. Then take responsibility and enjoy a healthy lifestyle. The lifestyle adjustments -- such as eating healthy foods and quitting smoking made today may reduce the risk of stroke tomorrow. ${ }^{22}$

\section{DIAGNOSIS OF STROKE}

Stroke Diagnosis is relatively straight forward, but it requires the rapid combination of medical personnel, technology, and at times, a little bit of luck so that all the testing can be done on time and appropriate treatment can be offered. Below you will find the main tests used by doctors during stroke diagnosis.

\section{The Neurological Examination}

This test is performed by a physician in order to uncover deficiencies in brain function which might confirm the suspicion that a person is actually having a stroke. Each part of the neurological exam tests a different area of the brain, including:

- $\quad$ Awareness and consciousness

- $\quad$ Speech, language, and memory function

- $\quad$ Vision and eye movements

- $\quad$ Sensation and movement in the face arms and legs

- $\quad$ Reflexes

- Walking and sense of balance

\section{COMPUTED TOMOGRAPHY SCAN}

This test is performed in the emergency room to detect a hemorrhagic stroke. Computed tomography (CT) scans are good tests for this purpose not only because they easily detect bleeding inside the brain, but also because they can be performed quickly. CT scans also can reveal ischemic strokes but only 6-12 hours after their onset. 


\section{Lumbar Puncture}

Also known as a "spinal tap" this test is sometimes performed in the emergency room when there is a strong suspicion for a hemorrhagic stroke in someone whose CT scan does not show clear blood. The test involves the introduction of a needle into an area within the lower part of the spinal column where it is safe to collect cerebrospinal fluid (CSF). When there is bleeding in the brain, blood can be seen in the CSF.

\section{Magnetic Resonance Imaging (MRI)}

This is one of the most helpful tests in the diagnosis of stroke because it can detect strokes within minutes of their onset. Its images of the brain are also superior in quality by comparison with CT images. Because of this, MRI is the test of preference in the diagnosis of stroke. A special type of MRI called magnetic resonance angiography,MRA, lets doctors precisely visualize narrowing or blockage of blood vessels in the brain.

\section{Transcranial Doppler (TCD):}

This test uses sound waves to measure blood flow through the major blood vessels in the brain. Narrow areas inside of a blood vessel demonstrate faster blood flow than normal areas. This information can be used by doctors to follow the progress of occluded blood vessels. Another important use for the TCD is the assessment of blood flow through blood vessels in the area of a hemorrhagic stroke, as these blood vessels have a propensity to undergo "vasospasm" a dangerous contraction of the wall of a blood vessel which can block blood flow.

\section{Cerebral Angiography:}

Stroke doctors use this test to visualize blood vessels in the neck and brain. During this test a special dye which can be seen using X-rays is injected into the carotid arteries, which bring blood to the brain. In a person who has a partial or a total obstruction in one of these blood vessels, or in any other blood vessel inside the brain, little or no dye can be seen flowing through it. A common cause of stroke is narrowing of a carotid artery, carotid stenosis, which is usually the result of cholesterol deposits along the walls of these blood vessels. This condition can also be diagnosed by a test called a Carotid Duplex, by which sound waves are used to evaluate blood flow through these blood vessels. Depending of the degree of narrowing and on the symptoms felt by a person, surgery might be needed to remove the plaque from the affected artery.

\section{Carotid Stenosis Treatments}

Cerebral angiography can also help doctors diagnose the following common conditions known to be associated with hemorrhagic stroke

- aneurysms,

- $\quad$ arterio-venous malformations

After a stroke is diagnosed, a new battery of tests needs to be performed in order to find out the cause of the stroke.

\section{Electrocardiogram}

This test, also known as an EKG or ECG, helps doctors identify problems with the electrical conduction of the heart. Normally, the heart beats in a regular, rhythmic pattern which promotes smooth blood flow towards the brain and other organs. But when the heart has a defect in electrical conduction, it stops beating rhythmically and it is said to be suffering from arrhythmia, or irregular heartbeats. Some arrhythmias, such as atrial fibrillation. Cause the formation of blood clots inside the heart chambers. These blood clots sometimes migrate to the brain and cause a stroke.

\section{Transthoracic echocardiogram (TTE)}

This test, also known as an 'echo' uses sound waves to look for blood clots or other sources of emboli inside the heart. It also is used to look for abnormalities in heart function which can lead to blood clot formation inside the heart chambers. TTEs are also used to investigate if blood clots from the legs can travel through the heart and reach the brain. 


\section{Leg Ultrasound}

Doctors usually perform this test on stroke patients diagnosed with a patent foramen ovale. The test uses sound waves to look for blood clots in the deep veins of the legs, which are also known as deep venous thromboses or DVTs. DVTs can cause strokes by making a long journey which ends up in the brain. First, a small fragment of a DVT breaks off and travels to the heart via the venous circulation. Once in the heart the blood clot crosses from the right side to the left side of the heart via the PFO, where it is propelled out via the aorta and carotids towards the brain, where it can cause a stroke.

\section{Blood Tests}

For the most part, blood tests help doctors look for diseases known to increase the risk of stroke, including:

- High cholesterol

- Diabetes

- Blood clotting disorders

\section{TREATMENT}

The treatment of stroke is given by following way

1) Surgical Treatment

2) Drug Therapy

3) Rehabilitation Therapy

\subsection{Surgical Treatment}

Several new surgical techniques for patients with arteriovenous malformations_and aneurysms: stereotactic microsurgery, cerebral revascularization and hypothermia. Stereotactic radiosurgery and interventional neuroradiology are also offered. The Stroke Center emphasizes acute intervention for optimal results.Surgery is an accepted way of preventing stroke for patients with certain conditions. A number of conventional surgical techniques have been in use for some time, including "clipping" aneurysms to prevent further bleeding and removing AVMs.Clinicians at the Stanford Stroke Center are pioneering several new surgical techniques for patients with AVMs or aneurysms once considered impossible to treat because of their location or size.

\subsection{2- Carotid Endarterectomy}

Carotid endarterectomy is used to remove atherosclerotic plaque from the carotid artery when this vessel is blocked. It has recently been proven that for certain patients with minor strokes or TIAs, carotid endarterectomy is highly beneficial in preventing future strokes. This procedure is also beneficial for some patients with blockage of the carotid arteries who have not had previous symptoms.

\subsection{3- Stereotactic Microsurgery for AVMs and Aneurysms}

Stereotactic microsurgery is one of the most dramatic new surgical procedures for AVMs and certain aneurysms that were once considered untreatable. It employs sophisticated computer technology to pinpoint the precise location of the AVM. This technique allows neurosurgeons to locate the AVM within one or two millimeters so they can operate, using microscope-enhanced methods and delicate instruments, without affecting normal brain tissue.

\subsection{4- Stereotactic Radiosurgery for AVMs}

Stereotactic radiosurgery is a minimally invasive, relatively low-risk procedure that uses the same basic techniques as stereotactic microsurgery to pinpoint the precise location of the AVM. Once located, the AVM can be obliterated by focusing a beam of radiation that causes it to clot and then disappear. Due to the precision of this technique, normal brain tissue usually is not affected. The procedure is generally performed on an outpatient basis.

\subsection{5- Hypothermia}

During surgical treatment of aneurysms and AVMs, there is a small risk that the patient may have a stroke while on the operating table. Stanford physicians are using a technique known as hypothermia (cooling of the body), to prevent stroke during surgical treatment of giant and complex aneurysms or difficult AVMs. Dropping the brain temperature gives the surgeon the necessary time to operate with minimal risk of surgeryinduced stroke. Special equipment known as a cardiopulmonary bypass machine is sometimes used to completely shunt blood flow away from the brain while the body is placed under deep hypothermia. 


\subsection{6- Revascularization of the Blood Supply}

Revascularization is a surgical technique for treating aneurysms or blocked cerebral arteries.The technique essentially provides a new route of blood to the brain by grafting another vessel to a cerebral artery or providing a new source of blood flow. Stanford is at the forefront of advances in revascularization techniques.

\subsection{7- Interventional Neuroradiology Techniques}

In addition to new medications and surgical techniques, the Stanford Stroke Center is pioneering a number of new interventional radiology procedures to prevent stroke in patients with selected high-risk AVMs, aneurysms, and partially blocked arteries. These endovascular procedures are performed within the blood vessel.

\subsection{8- Endovascular Treatment of Aneurysms}

Endovascular treatment of aneurysms is a new interventional neuroradiologic technique that greatly benefits patients with serious medical conditions who are unable to sustain the stress of surgery. Platinum coils developed at Stanford are guided into the aneurysm via a catheter, creating a clot that effectively closes the aneurysm off from the surrounding circulation, preventing the risk of hemorrhagic stroke in the future.

\subsection{9- Endovascular Treatment of AVMs}

Endovascular treatment of AVMs is also available at Stanford. One innovative form of treatment involves use of a "super glue" substance introduced via a tiny catheter to reduce the size of the AVM and facilitate further microsurgical or radiation treatment. In some cases, it is possible to completely block off and cure the AVM with endovascular treatment alone.

\subsection{0- Angioplasty and Stenting of Vessels in the Neck and Brain}

Angioplasty and stenting of vessels in the neck and brain are other new endovascular procedures available at only a few institutions nationwide. Cerebral angioplasty is similar to a widely used cardiology procedure and is used to open partially blocked vertebral and carotid arteries in the neck, as well as blood vessels within the brain. Stenting of carotid or vertebral arteries and large cerebral veins involves use of a fine, tubular wire mesh to hold the vessel open.

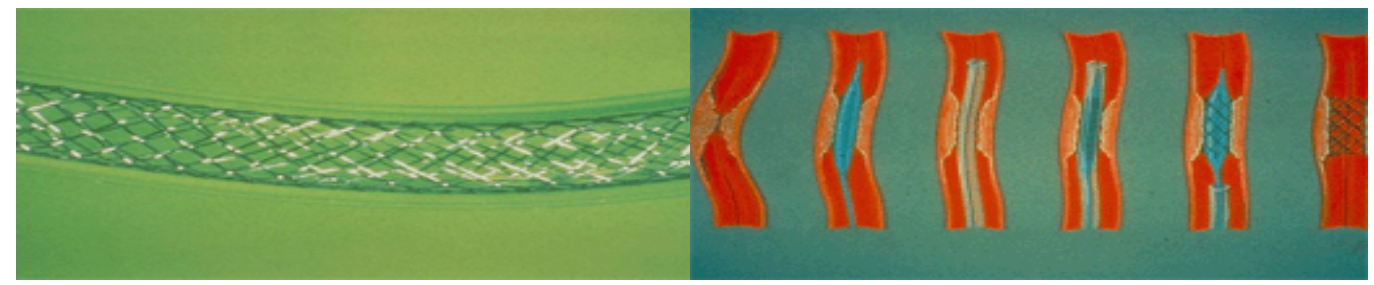

\section{2- Drug Therapy}

“Fig 8": Opening of Blood Vessel

\section{Herbal drug therapy Ginkgo Biloba}

Ginkgo biloba is used both to prevent and treat stroke. It helps to prevent blood clots from developing and increases blood flow to the brain. This herb has also been shown to inhibit free-radical formation.Ginkgo is widely used in Europe to treat complications of stroke, including memory and balance problems, vertigo and disturbed thought processes. Many studies show that this herb increases blood flow to the brain.Ginkgo also helps reduce fragility of the capillaries. (Capillaries are the tiny blood vessels that fan throughout your body.) This can help prevent hemorrhagic stroke.European physicians often prescribe an extract of ginkgo leaves for stroke survivors. Many doctors say that ginkgo prevents the stroke and, more importantly, it is nontoxic.

Dosage: Select a product containing at least 24 percent ginkgo heterosides (sometimes called flavoglycosides) and take 40 to 80 milligrams three times daily.

Note: Seek medical advice before taking ginkgo if you are regularly taking any type of blood-thinning medication, including aspirin. In large amounts, ginkgo may cause diarrhea, irritability and restlessness. ${ }^{25}$ 
Garlic

Garlic helps prevent ischemic stroke in three ways:

- Garlic reduces blood pressure

- Garlic lowers cholesterol levels

- Garlic is an anticoagulant.

Garlic is the best anti-clotting herb. It contains nine anticoagulant compounds. It is a major herb for heart attack prevention because of its blood-thinning effect and its ability to help control high blood pressure. These same effects also help prevent ischemic stroke. Well- researched studies show that taking garlic (raw or as a deodorized oil capsule) dramatically reduces platelet adhesiveness allowing improved circulatory function.Use more garlic in your cooking. You can also add it in your salad. (If you are not going for an important meeting right after that!) You can also take garlic capsules or aged garlic (preferred.). Onions, scallions, leeks, chives, and shallots have similar benefits.

Dosage: Take 500 milligrams three times daily.

Caution: If you have hemorrhagic stroke, stay away from garlic and its other anticlotting herbal relatives. (Ask your doctor whether you have ischemic or hemorrhagic stroke.)

Ginger (Zingiber officinale).Ginger is a cardiac tonic, as it decreases cholesterol and helps poor circulation. Ginger prevents blood from clotting excessively. In one Indian study, taking about two teaspoons of ginger a day for a week neutralized the blood-clotting effect of 100 grams of butter.You can use ginger in cooking, or you can brew ginger tea using one to two teaspoons of fresh grated root per cup of boiling water. Steep until cool. You can also sprinkle it in salads. It is a versatile herb.

\section{Turmeric (Cucurma longa)}

Many studies show that the compound curcumin, which is found in turmeric, helps prevent the formation of blood clots. Turmeric is an important herb in Ayurveda. It is also a key ingredient in Indian cooking and can be found in most curry spice blends. You might consider eating more curry dishes. You can find several recipes in our Healthy Recipes Section.

Carrot

In a Harvard study of 87,245 female nurses, consumption of carrots (and to a lesser extent, spinach) significantly reduced stroke risk. Women who ate five servings of carrots a week suffered 68 percent fewer strokes than those who ate carrots less than twice a month.Carrots are rich in beta-carotene and other carotenoids, all members of the vitamin A family. Other studies show that people can reduce their risk of stroke by as much as 54 percent if they eat lots of fruits and veggies that are rich in beta-carotene and vitamins $\mathrm{C}$ and E.So, eat more carrots. They are wonderful as snacks (especially the baby carrots). Include them in vegetable soups. You can also make carrot juice. If you really want to do it right, add some garlic and ginger to provide them with zest.

\section{Pigweed (Amaranthus)}

A six-year Harvard study of more than 40,000 health professionals showed that compared with those who consumed the least calcium, those who got the most had just one-third the risk of succumbing to heart attack. Many experts think that this also applies to stroke because of the biological similarities between ischemic strokes and heart attack.Pigweed is an excellent plant source of calcium, with 5.3 percent on a dry-weight basis. About one-third of an ounce of fresh pigweed leaves would provide 500 milligrams of calcium. (The Daily Value is 1,000 milligrams.)Use the young leaves in salads or steam the more mature leaves like spinach.

\section{Spinach}

Studies at Tufts University in Boston and the University of Alabama in Birmingham have demonstrated that folate can help prevent both heart disease and stroke. Compared with people who consumed little folate, those who ingested the most were only half as likely to show narrowing of the carotid artery, the artery that leads to the brain. Spinach, cabbage, endive, asparagus, papaya, okra and pigweed have folate Spinach is another vegetable found valuable in the prevention and treatment of stroke. The protection seems to come partly from beta carotene in carrots and spinach. A previous Harvard study found that eating the extra beta carotene in about one and a half carrots, which equals $200 \mathrm{~g}$ of mashed sweet potatoes or $170 \mathrm{~g}$ spinach (weighed raw and then cooked) every day saved 40 per cent off stroke rates. The drop was evident in those who 
ate $15-20 \mathrm{mg}$ of beta carotene daily compared to those who ate only $6 \mathrm{mg}$. The antistroke activity in carrots, spinach and other carotene-rich vegetables seems to emanate from their antioxidant properties

\section{English pea (Pisum sativum), Scurfy pea (Psoralea corylifolia)}

Nearly all legumes contain genistein, a cancer-preventive nutrient. In addition to guarding against cancer, genistein also appears to have a significant anti-clotting effect. So, it may also help prevent ischemic stroke and heart attack.You can get genistein from tofu and soy products. Or, you may choose English peas or other beans and legumes, instead.One food that is very rich in genistein is scurfy pea. This is consumed as a food (and reputed to be an aphrodisiac) in Asia. It contain much more genistein than soybeans!

\section{Willow}

Willow bark is herbal aspirin, and a low-dose aspirin has been shown in several studies to reduce the risk of ischemic stroke by about 18 percent. (Low-dose aspirin also cuts heart attack risk by about 40 percent in men and 25 percent in women.) Instead of taking aspirin, you can take a tea made from willow bark, meadowsweet or wintergreen. Add 1 or 2 teaspoons of any of these dried herbs to either hot herbal teas or cold lemonade. Drink two to three cups a day.

Caution: Willow bark and the other aspirin-like herbs should only be used to prevent and treat ischemic stroke. They are powerful anticoagulants. Hence, they may increase risk of hemorrhage, including hemorrhagic stroke. If you're at risk for this type of stroke, consult your doctor before taking aspirin or any aspirin-like herbs. Avoid these if you're allergic to aspirin.

\section{Pineapple}

Pineapple contains an enzyme known as bromelain that is best known for its ability to break down proteins. It's a key ingredient in meat tenderizers. But bromelain also has an anti-clotting action that might help prevent ischemic stroke and heart attack.

\section{Bilberry (Vaccinium myrtillus)}

Bilberries, blueberries and huckleberries contain compounds known as anthocyanidins. European studies show that these compounds help prevent blood clots and also break down plaque deposits lining the arteries. Bilberries are also shown to help to maintain capillaries. Bilberries and their relsatives might help prevent ischemic stroke without increasing the risk of hemorrhagic stroke. One glass of huckleberry juice taken twice a week may help prevent stroke, according to herbalists.

\section{Evening primrose}

Evening primrose oil is rich in gamma-linolenic acid (GLA), which has potent anti-clotting and blood pressurelowering actions. It is believed to be useful in the prevention of stroke and heart disease. Borage oil is also rich in GLA.

Astragalus :Astragalus improves tissue oxygenation.

Dosage: Take 250 milligrams of standardized extract four times a day.

Calamus :Calamus helps restore brain tissue damaged by stroke.

Dosage: Standard infusion 3-9 g; Tincture - 10-30 drops.

Cayenne Pepper :Cayenne pepper improves circulation and heart function without raising blood pressure. It also enhances the power of other herbs taken at the same time.

Dosage: Take 100 milligrams twice daily, with meals.

Green Tea :Green tea may act as one of the most potent free-radical scavengers to protect against the peroxidation of lipids, a contributing factor in atherosclerosis.

Choose a decaffeinated standardized extract containing at least 50 percent catechins and 90 percent total polyphenols,

Dosage: Take 300 to 500 milligrams daily.

\section{Hawthorn}

Hawthorn has been reported to prevent or slow the progression of arteriosclerosis.

Dosage: Choose a standardized extract containing 1.8 percent vitexin-2 rhamnosides and take 100 to 200 milligrams two or three times a day.

Horsetail :IThe silica in horsetail maintains the elastic connective tissue of the arteries. It promotes arterial impermeability to harmful lipids, preventing deposits.

Dosage: Take 1 cup of horsetail tea or 1 tbsp. of horsetail juice three times daily. 
Kava kava : Kava kava helps to protect the brain against oxygen deprivation.

Dose: Choose a standardized extract containing 30 percent kavalactones and take 250 milligrams twice a day. Note: In excess amounts, this herb can cause drowsiness. Do not exceed the recommended dose. Do not use kava kava if you are pregnant or nursing, if you have Parkinson's disease, or if you are taking a prescription medication for depression or anxiety.

\section{Spine-bark and Grape-seed Extract}

Pine-bark and grape-seed extract are high in proanthocyanidins (also known as OPCs) that increase the structural strength of weakened blood vessels.

Dosage: Take 25 to 50 milligrams of either two or three times daily.

II) Homeopathic drug therapy

Homeopathic Remedies for Stroke :Homeopathic treatments are intended to support recovery from a stroke, rather than to treat an acute episode. If you suspect you may be having a stroke, seek emergency medical treatment immediately.

First aid for stroke, if the person loses consciousness:

1) Aconite 30c: if the person is very fearful

2) Opium 6c: if the person has collapsed; face is dark and flushed; loud, "snoring" breathing; cheeks puff out as person exhales

3) Arnica 6c: Once the person's condition is stable, give every 4 hours for up to 3 days.

\section{In case of emergency,}

give one of the following strength every fifteen minutes for up to ten doses during a stroke while waiting for medical assistance. You should call for medical assistance immediately.

1) Belladonna 30c: if the person's face is hot and flushed, if there is headache, if the eyes are wide and staring

2) Nux vomica 6c: at first signs of attack, especially if brought on by a heavy meal or alcohol

3) Aconite 30c: for a person who is panicky and afraid of dying once he or she realizes what is happening

4) Opium 6c: In later stages, if the person is lapsing into consciousness, face is bluish and congested, and breathing is heavy and labored, take four times daily for up to two weeks immediately after a stroke.

5) Arnica $6 \mathrm{c}$ to be taken four times daily for up to three weeks during recovery.

6) Baryta 6c: if the person is elderly and physically and mentally weak.

7) Gelsemium 6c: where main aftereffects are numbness and trembling, in- ability to speak, pain at back of head.

8) Lachesis 6c: if speech is very slow.

9) Hyoscyamus 6c: if speech is unintelligible, and person has a tendency to clutch private parts.

10) Aurum 6c: if the person is clearly depressed.

After Your Stroke :The best approach to treating the aftermath of stroke homeopathically is to consult a qualified homeopathic practitioner who can prescribe a constitutional remedy. The remedies that follow can be helpful as well.

\section{1) Aconite and Arnica}

Aconite can help alleviate the panic, fright, and shock that accompany a stroke.

Arnica helps to initiate bodywide healing.

Dose: As soon as your doctor signals that you can take something by mouth, take one dose of Aconite 200x or 30c. Follow this with one dose of Arnica 30x or 15c three to four times daily for three to five days.

2) Aurum muriaticum is good if your predominant symptom is depression. Take one dose of Aurum muriaticum 30x or $15 \mathrm{c}$ three times daily for up to three days, as needed. If you notice an improvement before that time, stop taking the remedy.

3) Baryta carbonica is helpful for both physical and mental weakness and fatigue following a stroke. Take one dose of Baryta carbonica 30x or 15c three times daily for up to three days, as needed. If you notice an improvement before that time, stop taking the remedy.

4) Gelsemium can help if you have numbness and trembling, and have lost the ability to speak. Take one dose of Gelsemium 30x or 15c three times daily for up to three days, as needed. If you notice an improvement before that time, stop taking the remedy. 


\subsection{Medicinal Treatment Antiplatelets}

Platelets are blood cells that help the blood to clot and prevent bleeding. When the body has a cut, scratch, bruise or bleed, platelets go into action and begin to work. These platelet cells need thromoxane A2 and adenosine; vitamin K specific clotting factors (chemicals produced by the body) to make them stick together. These chemicals are essentially the glue that holds the blocks together to make the clot. However, in patients who have had a TIA or stroke, the blocks don't need to stick together as much because this causes the blood to be too thick (like adding flour to milk when making a cake batter) and possibly form a clot that can't fit through the vessels.Doctors often place stroke and TIA patients on blood thinners to decrease the possibility of the body forming another clot in the blood, which may lead to another stroke and TIA. Below is information on specific types of antiplatelets used for stroke prevention.

Aspirin (acetylsalicylic acid, ASA)Aspirin: Besides relieving pain, fever and inflammation, aspirin has many other uses. Aspirin is also used as an antiplatelet/platelet aggregation inhibitor (to keep your blood from sticking together) in patients who have had a TIA or stroke. It can also reduce the risk of having another TIA or stroke. Different doses are used for this purpose, ranging from $50 \mathrm{mg}$ to $325 \mathrm{mg} /$ day, depending on the patient's condition and the doctor's decision. The idea that "if one is good for me, two or three must be better" is wrong. Do not adjust your dose without first talking to the pharmacist or doctor that dispensed or prescribed the medication. The dose is not the same for everyone. The usual dose for stroke/TIA prevention is $30-325 \mathrm{mg}$ a day. Take the medication according to the prescription. Even though you may be taking an 81mg (baby aspirin) a day, someone else who had a stroke may be taking $325 \mathrm{mg}$ of aspirin a day or may be on a different medicine for stroke prevention. Don't rely on what you hear from other stroke patients, and don't take more or less of the drug without first talking to the doctor who prescribed it.Since aspirin can irritate the stomach, it is best to take it with food or a full glass of water or milk to help avoid or lessen possible stomach problems. Aspirin will help prevent platelets from making the blood too thick, thereby reducing the risk of a clot. Taking aspirin regularly will lessen your body's ability to stop bleeding when you are cut, scratched, or bruised. Your doctor wants the aspirin to thin the blood (decrease the body's ability to form a clot) just enough to help prevent a future TIA or stroke. Watch for blood in the urine, stools, or around the gums when eating and brushing teeth; bleeding from the nose; or easy bruising. If you notice abnormal or excessive bleeding, let your pharmacist and doctor know, and talk with them before you change or take any new medicines. Abdominal (stomach area) pain that will not go away and ringing in the ears are other signs that there might be a problem with the medication. Let the pharmacist or doctor know if you have any of these warning signs.Talk with your pharmacist or doctor before taking any other aspirin products, anti-inflammatory agents (Aleve, Naprosyn, Ibuprofen, etc...) or any other medications.Clopidogrel

Plavix : Clopidogrel is an antiplatelet/platelet aggregation inhibitor drug that is used to help prevent another stroke. It does this by decreasing the blood's ability to clot. This means that when you get a cut or scratch it will take a little longer to stop bleeding. Watch for blood in the urine, blood in the stools, bleeding around the gums when eating and brushing teeth, bleeding from the nose, or bruising easily. If you notice abnormal or excessive bleeding, let your pharmacist and doctor know, and talk with them before you change or take any new medicines.You will take one $75 \mathrm{mg}$ tablet once a day in the morning (can be taken with or without food), or when and how your pharmacist and doctor tell you. Do not adjust the dose without first talking to the pharmacist or doctor that dispensed or prescribed the mediation, and be sure to talk with your pharmacist or doctor before taking any other medications.

\section{Dipyridamole}

Aggrenox :Aggrenox is the combination of aspirin $(25 \mathrm{mg}$ ) and extended release dipyridamole (200 $\mathrm{mg}$ ), two antiplatelet/platelet aggregation inhibitors. Each of these medications work together in similar ways but on separate chemicals in the blood to help prevent a future TIA or stroke. Do not adjust the dose without first talking to the pharmacist or doctor who dispensed or prescribed the medication. The aspirin portion of the combination works in the same way as above, but the dirpyridamole helps prevent platelets, blood cells and the vessels from using adenosine (another chemical that helps the bricks and glue form a clot). Dipyridamole also has the potential to vasodilate the vessels that carry the blood to allow more blood and particles to flow through (like a water hose expanding in the summer sun). The combination capsule Aggrenox (aspirin and dipyridamole) cannot be substituted by taking each drug separately. Taking the two separately does not have the desired effect as the combination capsule does. Take one capsule in the morning (with or without food) and take one capsule in the evening (with or without food) for a total of two capsules a day. Swallow the capsule. Do not chew it or crush it. It must be swallowed whole. Take the medication the way the pharmacist and doctor told you. 
Many patients starting on Aggrenox develop a severe headache due to the vessels in the sbrain vasodialating (expanding). This headache tends to decrease and go away as the body gets used to the medicine. In the meantime, use an over-the-counter pain reliever and call the pharmacist or doctor to inform them of the headache.The combination of aspirin and dipyridamole is a stronger blood thinner than either drug alone. So, watch for blood in the urine, blood in the stools, bleeding around the gums when eating and brushing teeth, bleeding from the nose, or bruising easily. If you notice abnormal or excessive bleeding, let your pharmacist and doctor know, and talk with them before you change or take any new medicines. Talk with your pharmacist or doctor before taking any other aspirin products, anti-inflammatory agents (Aleve, Naprosyn, Ibuprofen, etc...) or any other medications.

\section{Ticlopidine}

Ticlopidine is used to help prevent another stroke. You will take a 250mg tablet two times a day (one in the morning and one in the evening) with food. Take the medicines like the pharmacist and doctor told you, and do not adjust the dose without first talking to the pharmacist or doctor that dispensed or prescribed the medications. Be especially observant of any excessive bleeding such as blood in the urine, blood in the stools, bleeding around the gums when eating and brushing teeth, bleeding from the nose, or bruising easily. If you notice abnormal or excessive bleeding, let your pharmacist and doctor know, and talk with them before you change or take any new medicines. You will have to have lab work done every two weeks for the first three months of treatment to check your blood levels. Talk with your pharmacist or doctor before taking any other aspirin products, anti-inflammatory agents (Aleve, Naprosyn, Ibuprofen, etc...) or any other medications.

Thrombolytic :Thrombolytic therapy is the use of drugs to break up the clot that is causing the disruption of blood flow to the brain. It is critical that you immediately go to the hospital when you first notice the warning signs of a stroke. The length of time between the first warning signs and the time you get to a hospital may be the difference between a good or poor outcome. Patients who present to the hospital within 3 hours of the first sign of a stroke may be able to receive alteplase (tPa, Activase Alteplase is a clot-buster that can restore blood flow to the area of the stroke.

Tissue Plasminogen Activator (tPA, Activase $\left.{ }^{\circledR}\right)$ :Tissue plasminogen activator is a thrombolytic drug (a "clotbuster"). It can reduce the severity of ischemic stroke if it is given within three hours of stroke onset. This drug can be given intravenously or by arterial catheter, but not by mouth. In patients who present with stroke symptoms within 3 hours of onset and have no evidence of hemorrhage or infarct size greater than one third of the middle cerebral artery territory on CT brain, Treatment with IV recombinant tissue plasminogen activator (rtPA) is recommended at a dose of $0.9 \mathrm{mg} / \mathrm{kg}$ (maximum dose, $90 \mathrm{mg}$ ) over 1 hour, with the first $10 \%$ given as a bolus over 1 minute (Class I, Level A evidence). Studies have demonstrated that $31 \%$ to $50 \%$ of patients treated with rtPA experienced improved recovery at 3 months as compared to $20 \%$ to $38 \%$ of patients in the placebo arm. However, strict adherence to national guidelines in the administration of rtPA and postlysis management is critical, given the $6 \%$ risk of intracranial hemorrhage.

Anticoagulants :Anticoagulants are a class of drugs commonly used to prevent the blood from forming dangerous clots that could result in a stroke. Often called "blood thinners," anticoagulants are often the first medication prescribed by doctors following a stroke. By reducing the ability of the blood to clot and thereby reducing the likelihood of coronary or vascular emboli anticoagulants are frequently used in patients who are already at high-risk for stroke. Below is information on specific types of anticoagulants used for stroke prevention.

Heparin :Heparin can be given intravenously or sub-cutaneously, but not by mouth. Heparin is sometimes used to reduce acute stroke damage or stroke risk in hospitalized patients. In addition, heparin may be used in hospitalized stroke patients to reduce the risk of blood Sclots forming in leg veins.

\section{Warfarin (Coumadin ${ }^{\circledR}$ and others)}

Warfarin is an anticoagulant drug ("blood thinner") which is taken by mouth. Daily use of warfarin can reduce the risk of stroke in certain patients. For example, many patients with atrial fibrillation (an irregular heartbeat) should be prescribed warfarin. Use of warfarin requires careful monitoring, and you should closely follow your doctor's recommendations, including regular blood tests. Let your doctor know if you're taking any other medications. Your doctor will start you on a low dose of Coumadin and will have you see him or someone else to check your blood weekly. You want your blood levels to be within a certain range. The doctor wants you to be right in the middle, so you will have your blood drawn and the doctor will increase or decrease your dose based on the blood values and where he or she wants you to be. 
Patients receiving Coumadin also need to be very careful about their diet and activities to prevent problems while taking the medication. The levels of the drug in the body can be affected by the amount of vitamin $\mathrm{K}$ in your diet. Foods high in vitamin $\mathrm{K}$ include leafy green vegetables, green teas, as well as pork and beef liver. Patients should avoid large amounts of alfalfa, broccoli, asparagus, brussels sprouts, cauliflower, cabbage, kale, spinach, watercress, lettuce and turnip greens. You can still eat these foods, but eat the same amount regularly. For example, don't eat a plateful of turnips every day for a week and then decide to stop eating them the next week. Large changes in the amount you eat can cause problems with your treatment.Also, since Coumadin ${ }^{\circledR}$ is a fairly strong blood thinner, you have a chance of bleeding more than usual with common cuts, scrapes and falls. Use caution walking and with activities that place you at risk to fall or get hurt. Be careful while shaving, because a common cut may take longer to stop bleeding. Watch for blood in the urine, in the stools, or around the gums when eating and brushing teeth; bleeding from the nose; or bruising easily. If you notice abnormal or excessive bleeding, let your pharmacist and doctor know, and talk with them before you change or take any new medicines.

\section{Tenecteplase faster, more effective at cerebral artery recanalization than tPA}

Tenecteplase (TNK), a new, modified form of tissue plasminogen activator (tPA), is faster, more effective, safer and easier to administer than alteplase (Activase, Genentech Inc., South San Francisco, CA), the only currently approved form of tPA.Those findings come from the Spanish TNK-TPA Reperfusion Stroke Study, and were presented by Dr. Carlos A. Molina of Hospital Vall d'Hebron in Barcelona during the American Stroke Association's International Stroke Conference 2008. The study involved 122 consecutive patients with acute ischemic strokes due to middle cerebral artery (MCA) occlusion and eligible for thrombolytic therapy. All patients had a diffusion-weighted/perfusion-weighted (DWI/PWI) MRI mismatch of more than 20 per cent of the DWI lesion, indicating a large area of ischemia surrounding the infarcted penumbra of the lesion.Patients were randomized to a $0.4 \mathrm{mg} / \mathrm{kg}$ bolus of tenecteplase or standard tPA $0.9 \mathrm{mg} / \mathrm{kg}$, with the first ten per cent given as a bolus and the remainder in a one-hour infusion). One-third of patients received tenecteplase and twothirds received standard tPA.Complete recanalization at two hours occurred in 42.4 per cent of the tenecteplase group and in 33.4 per cent of standard tPA-treated patients. Some degree of MCA recanalization at two hours took place in 69 per cent of the tenecteplase group and 53 per cent of the standard tPA group. The beginning of recanalization occurred at 19 minutes in the tenecteplase group and 24 minutes with standard tPA, which was not a statistical difference. However, the difference in recanalization rates at two hours did reach statistical significance."There is a modest advantage (with tenecteplase) over standard tPA in opening blood vessels."Asymptomatic intracranial hemorrhage was seen on CT at 24-36 hours in 28 per cent and 21 per cent of the tenecteplase and alteplase groups, respectively.NIHSS scores at 24 hours had improved by more than four points in 63 per cent of the tenecteplase-treated patients and in 51 per cent of alteplase-treated patients.Clinical recovery was 2.5 -fold greater with the newer modified form of tPA than standard tPA.Functional independence at three months occurred in 66 per cent of tenecteplasetreated and 52 per cent of standard tPA-treated patients. Tenecteplase has a longer half-life and it is easier to administer than standard tPA, the Spanish investigator noted. These phase 2 results "merit direct, randomized phase 3 trials,"

\subsection{Stroke Rehabilitation Therapy \\ Stroke Rehabilitation Programs}

Following a stroke, your life may not return to normal. Strokes cut off oxygen to areas of your brain controlling everything from basic movements to speech, which leaves many stroke victims needing regular rehabilitation programs.

\section{Outpatient Rehabilitation for Strokes}

A stroke is a life-changing event that requires emergency treatment initially and extended medical and rehabilitative care afterword. Rehabilitation typically begins in the hospital. But because it can take months or even years.

Muscle Relearning in Stroke Rehabilitation:Stroke rehabilitation may include muscle relearning technique in situations where a stroke has caused reduced movement or partial paralysis. The success of rehabilitation following a stroke has many variables, such as the cause.

Topics in Stroke Rehabilitation:Depending on the severity and anatomy of the stroke, the intensity of these changes will vary. However, the constant, regardless of stroke type, remains that a patient's life changes physically, cognitively and emotionally. 
How to Rehabilitation the Tibialis Muscle After a Stroke :These muscles also help stabilize the arch of your foot and help slow down the rotation of your foot when performing activities like walking and running. Following a stroke, you may experience a loss of motor control on one side.

Hydrotherapy for Stroke Rehabilitation :Exercise can be a valuable tool in rehabilitation for stroke survivors, and hydrotherapy is particularly helpful for the weakest individuals. Water exercise isn't a cure for a stroke, but it can help improve strength, mobility.

Stroke Rehabitation at Home :It can cause severe damage to a patient's physical and mental capabilities. Fortunately, proper rehabilitation can help restore cognitive, communication and physical skills that were impaired by the stroke.

Rehabilitation for Hand Paralysis After a Stroke :The damage associated with a stroke can affect any part of your body, including your hands. With the help of an appropriate rehabilitation program, you may be able to overcome the effects of a stroke and regain at least some hour.

Stroke Rehabilitation \& Physiotherapy :This cerebrovascular event is a leading cause of death worldwide. If you have experienced a stroke, rehabilitation is important to finding your way back to some level of independence and normalcy. Althoug many methods are used...

\section{Muscle Relearning in Stroke Rehab With Electrical Stimulation}

By providing a shock to specific muscles or muscle groups, ES allows the stroke survivor to better utilize affected arms or legs to perform motor skills. Physical and occupational therapists use ES as part of poststroke rehabilitation.

\section{Exercise to Improve a Rehabilitation Patient's Coordination}

Exercise is a crucial element of successful rehabilitation, regardless of the cause of injury. Not only does exercise strengthen and tone the muscles, but it also improves coordination, which is often one of the primary areas. ${ }^{[29],[30],[31]}$

\section{Prevention}

Stroke Prevention Guidelines

Up to 80 percent of all strokes can be prevented start reducing risk now.

Although stroke can happen to anyone, certain risk factors can increase chances of a stroke. However, studies show that up to 80 percent of strokes can be prevented by working with a healthcare professional to reduce personal risk. It is important to manage personal risk and know how to recognize and respond to stroke signs and symptoms. Learn interactively about more than 20 leading risk factors for stroke through the interactive risk factor tool.The following Stroke Prevention Guidelines will help you learn how you may be able to lower your risk for a first stroke. ${ }^{25}$

National Stroke Association's Stroke Prevention Advisory Board, an elite group of the nation's leading experts on stroke prevention, established the first Stroke Prevention Guidelines. They were published in a 1999 issue of Journal of the American Medical Association (JAMA) and have been updated to reflect current medical standards.Talk to a healthcare professional and follow these guidelines.

\section{Know blood pressure (hypertension)}

High blood pressure is a major stroke risk factor if left untreated. Have blood pressure checked yearly by a doctor or at health fairs, a local pharmacy or supermarket or with an automatic blood pressure machine.

\section{Identify atrial fibrillation (Afib)}

Afib is an abnormal heartbeat that can increase stroke risk by 500\%. Afib can cause blood to pool in the heart and may form a clot and cause a stroke. A doctor must diagnose and treat Afib.

\section{Stop smoking}

Smoking doubles the risk of stroke. It damages blood vessel walls, speeds up artery clogging, raises blood pressure and makes the heart work harder. 


\section{Know cholesterol levels}

Cholesterol is a fatty substance in blood that is made by the body. It also comes in food. High cholesterol levels can clog arteries and cause a stroke. See a doctor if your total cholesterol level is more than 200.

\section{Control diabetes}

Many people with diabetes have health problems that are also stroke risk factors. A doctor and dietician can help manage diabetes.

\section{Manage exercise/diet}

Excess weight strains the circulatory system. Exercise five times a week. Maintain a diet low in calories, salt, saturated and trans fats and cholesterol. Eat five servings of fruits and vegetables daily.

\section{Treat circulation problems}

Fatty deposits can block arteries carrying blood to the brain and lead to a stroke. Other problems such as sickle cell disease or severe anemia should be treated.

\section{Transient Ischemic Attack (TIA)}

A TIA is a temporary episode of stroke-like symptoms that can last a few minutes to 24 hours but usually causes no permanent damage or disability. TIA and stroke symptoms are the same. Recognizing and treating a TIA can reduce stroke risk. Up to 40 percent of people who experience a TIA may have a stroke ${ }^{[21]}$

\section{CONCLUSION}

It is very difficult to regenerate brain cells when they have died. There has been a lot of work done in this area and there are glimmers of hope. Strokes damage brain cells and the amount of damage and the severity can vary enormously. At one extreme it could be physical movement affected, but at the other memory and reasoning are lost. The final danger will always be the death of the victim from the effects of the stroke. Is it you? Keep calm! If you think you are having a stroke then you need emergency medical help now to limit the long-term effects of the stroke on your brain. You may not be understood if you call for emergency help. Your friends, family or co-workers may not understand you but it is urgent to get help now. Realize that once you have had one stroke then there is a danger that you will have another. Even if the first stroke was a warning or a mini-stroke they can never be taken lightly as the next one may be far more serious. Get medical help and get it now. Is it a friend, family member or co-worker? Maybe you don't recognize the symptoms. Maybe someone else has had a stroke but the symptoms may be completely different this time. One may have been paralyzed down one side, another may lose feeling or be unable to understand or speak properly. Get properly qualified help now. A first aider should recognize the symptoms and call for emergency help. If there is no first aider immediately available then call for the ambulance or paramedics yourself. Do not delay as the delay may be fatal. With someone having a stroke every 3-4 minutes in the US and every 5 minutes in the UK the likelihood is that this is a stroke and they need urgent medical help. The long haul starts here. It will take time to recover. In a later book we will be looking at after the stroke and how you can be helped to overcome the limitations of the disability they call a stroke. In the vast majority of cases, you will walk and talk and smile again. It may not be as good as you want it but you can work within those limitations and seek to limit the dangers of having another stroke by stopping smoking, changing your diet and getting exercise. If you are a smoker you are probably saying it is time all these do-gooders shut up and stopped nagging me to stop smoking. I enjoy it and I am not going to stop. Then realize that you have decided to put yourself in danger and see where it takes you. Your family is concerned for you and to clean up your clothes and their atmosphere. All of us can benefit from more exercise and a better diet. You should be eating less fats and far more healthy food. So take the right steps now to limit the danger to yourself and your family. If you or a member of your family suffers a stroke then you realize just how devastating it can be to suffer with it. Take the right steps now to limit your danger of being the next victim.

\section{ACKNOWLEDGEMENTS}

I express my special thanks to Dr. R. Y. PATIL Principal Shankarrao Ursal College of Pharmaceutical Sciences And Research Center, Kharadi, Pune-14. I am honoured to express my profound and deep sense of gratitude towards my respectable guide Mrs.P.N.JAGTAP.For his creative suggestions, helpful discussion, unfailing advice constant encouragement and kind support during the project work. 
I would like to express my gratitude to my parents who always supported and encouraged me. Thus, I have solemnized my participation in this project with dedication to make it a success.

\section{REFERENCES}

Adams HP Jr, etal. (2007). Guidelines for the early management of adults with ischemic stroke: A guideline from the American Heart Association/American Stroke Association Stroke Council, Clinical Cardiology Council, Cardiovascular Radiology and Intervention Council, and the Atherosclerotic Peripheral Vascular Disease and Quality of Care Outcomes in Research Interdisciplinary Working Groups: The American Academy of Neurology affirms the value of this guideline as an educational tool for neurologists. Stroke,

[2] American Heart Association. Heart disease and stroke statistics: 2005 update. Availableat: http://www.americanheart.org/downloadable/heart/ 1105390918119hdsstats2005update.pdf. Accessed June 14, 2005.

[3] Abraham J, Rao PSS, Inbaraj SG, Shetty G, Jose CJ. An epidemiological study of hemiplegia due to stroke in South India. Stroke 1970

[4] Bazzano LA, He J, Ogden LG, et al. Dietary intake of folate and risk of stroke in US men and women: NHANES Epidemiologic Follow-up Study. National Health and Nutrition Examination Survey. Stroke.2002;

[5] Fang J, Madhavan S, Alderman MH. Dietary potassium intake and stroke mortality. Stroke. 2000

[6] Khaw KT, Barrett-Connor E. Dietary potassium and stroke-associated mortality. A 12-year prospective population study. N Engl J Med. 1987;

[7] Strokes still killing us in epidemic proportions

a. Nov 10, 1998; Philadelphia Tribune, The 11-10-1998strokes still killing us in epidemic proportionsin Western countries, strokes...

[8] Atlas of heart disease and stroke, WHO, 2004 September Heart Association. Stroke

outcomes classification. Available at: http://www.americanheart.org/presenter.jhtml?Identifier=1859. Accessed May 30, 2005

[10] Barnett HJ, Gunton RW, Eliasziw M, et al. Causes and severity of ischemic stroke in patients with internal carotid artery stenosis. JAMA. 2000.

[11] Leppala JM, Virtamo J, Fogelholm R, Albanes D, Heinonen OP. Different risk factors for different stroke subtypes: association of blood pressure, cholesterol, and antioxidants. Stroke.1999

[12] Voko Z, Hollander M, Hofman A, Koudstaal PJ, Breteler MM. Dietary antioxidants and the risk of ischemic stroke: the Rotterdam Study. Neurology. 2003;61:1273-1275.

[13] The Stroke Association. Suspect a stroke? Act FAST. [Last accessed on: 28 May 2008]. Available from: The Stroke Association. Strokes common but signs are hard to recognize Jan 02, 1996; M.J. mccollum Philadelphia Tribune, The 01-02-1996 strokes common but signs are hard to recognize.stroke are the..

[14] J.P.Mohr, Dennis Choi, James Grotta, Philip Wolf (2004).Stroke: Pathophysiology, Diagnosis, and Management . Newyork: Churchill Livingstone.

[15] Lees KR, Zivin JA, Ashwood T, Davalos A, Davis SM, Diener HC, et al. NXY-059 for acute ischemic stroke. N Engl J Med. 2006;

[16] Wolf PA, Abbott RD, Kannel WB. Atrial fibrillation as an independent risk factor for stroke: the Framingham Study. Stroke. 1991;

[17] He K, Merchant A, Rimm EB, et al. Folate, vitamin B6, and B12 intakes in relation to risk of stroke among men. Stroke. 2004;

[18] Suk SH, Sacco RL, Boden-Albala B, et al. Abdominal obesity and risk of ischemic stroke: the Northern Manhattan Stroke Study. Stroke. 2003;

[19] Reynolds K, Lewis B, Nolen JD, Kinney GL, Sathya B, He J. Alcohol consumption and risk of stroke: a meta-analysis. JAMA. 2003;

[20] Klatsky AL, Armstrong MA, Friedman GD, Sidney S. Alcohol drinking and risk of hospitalization for ischemic stroke. Am J Cardiol. 2001;

[21] National Collaborating Centre for Chronic Conditions. Acute stroke and TIA: guideline methodology pack. London: NCC-CC; 2006.

[22] Charles P. Warlow, Jan van Gijn, Martin S. Dennis, Joanna M. Wardlaw, John M. Bamford, Graeme J. Hankey, Peter A. G. Sandercock, Gabriel Rinkel, Peter Langhorne, ICathie Sudlow,

PeterRothwell (2008). Stroke: Practical Management. 3rdedition, Wiley-Blackwell.

Stroke: From recognition by the public to management by emergency medical services

Jan 01, 2002; ABSTRACT To improve the outcomes of stroke patients, public awareness of stroke must be increased and emergency...

Www.holisticonline.com/remedies/Heart/stroke_alt_herbs.htm

BJ Quilliam, KL Lapane, C Leibson - Stroke, 2001 - American Heart Association...

F Gueyffier, F Boutitie, JP Boissel, S Pocock... - Annals of Internal ..., 1997 - ukpmc.ac.uk

Bates B, Choi JY, Duncan PW, Glasberg JJ, Graham GD, Katz RC, et al. Veterans affairs/department of defense clinical practice guideline for the management of adult stroke rehabilitation care: executive summary. Stroke. 2005

[28] Duncan PW, Zorowitz R, Bates B, Choi JY, Glasberg JJ, Graham GD, et al. Management of adult stroke rehabilitation care: a clinical practice guideline. Stroke. 2005;

[29] Kwakkel G, Wagenaar RC, Koelman TW, et al. Effects of intensity of rehabilitation after stroke: a research synthesis. Stroke. 1997;

[30] Adams RJ (2008). AHA/ASA science advisory: Update to the AHA/ASA recommendations for the prevention of stroke in patients with stroke and transient ischemic attack.

[31] Yochum LA, Folsom AR, Kushi LH. Intake of antioxidant vitamins and risk of death from stroke in postmenopausal women. Am J Clin Nutr. 2000;

[32] Berthold HK, Sudhop T. "Garlic preparations for prevention of atherosclerosis." Curr Opin Lipidol. 1998 